Published in final edited form as:

Cancer Immunol Res. 2013 October ; 1(4): 229-234. doi:10.1158/2326-6066.CIR-13-0020.

\title{
Increased frequency of ICOS+ CD4 T-cells as a pharmacodynamic biomarker for anti-CTLA-4 therapy
}

\author{
Derek Ng Tang1 ${ }^{1}$, Yu Shen ${ }^{2}$, Jingjing Sun ${ }^{1}$, Sijin Wen ${ }^{3}$, Jedd D. Wolchok ${ }^{4,5}$, Jianda Yuan ${ }^{5}$, \\ James P. Allison ${ }^{6}$, and Padmanee Sharma ${ }^{1,6, \dagger}$ \\ ${ }^{1}$ Department of Genitourinary Medical Oncology, The University of Texas M. D. Anderson Cancer \\ Center, Houston, TX 77030.
}

${ }^{2}$ Department of Biostatistics, The University of Texas M. D. Anderson Cancer Center, Houston, TX 77030.

${ }^{5}$ Department of Immunology, The University of Texas M. D. Anderson Cancer Center, Houston, TX 77030.

${ }^{3}$ Department of Biostatistics, West Virginia University Health Science Center, Morgantown, WV 26506.

${ }^{4}$ Department of Medicine, Memorial Sloan-Kettering Cancer Center, New York, New York 10065.

${ }^{6}$ Ludwig Center for Cancer Immunotherapy, Memorial Sloan-Kettering Cancer Center, New York, New York 10065.

\section{Abstract \\ Pharmacodynamic biomarkers can play an important role in understanding whether a therapeutic agent has "hit its target" to impact biologic function. A pharmacodynamic biomarker for anti- CTLA-4 therapy remains to be elucidated. We previously reported that anti-CTLA-4 therapy increases the frequency of CD4 T-cells expressing the inducible costimulator (ICOS) molecule. To determine whether the frequency of ICOS ${ }^{+} \mathrm{CD} 4 \mathrm{~T}$-cells could be used as a pharmacodynamic biomarker for anti-CTLA-4 therapy, we performed flow cytometric studies and statistical analyses on data from 56 individuals, which included 10 healthy donors, 36 patients who received anti- CTLA-4 monoclonal antibody (mAb), and 10 patients who received treatment with a different immunomodulatory agent (gp100 DNA vaccine). After treatment with anti-CTLA-4 mAb (ipilimumab, Bristol Myers-Squibb), we detected a statistically significant increase in the frequency of ICOS ${ }^{+} \mathrm{CD} 4 \mathrm{~T}$-cells. After two doses of anti-CTLA-4 therapy, the assay was found to have an estimated specificity of 96\% (95\% confidence interval: $88 \%-100 \%$ ) and sensitivity of $71 \%$ (95\% CI: $54 \%-85 \%$ ), with positive expression defined as a frequency that is greater than the upper bound of $95 \%$ confidence interval among baseline samples from all subjects. Our data suggest that an increased frequency of $\mathrm{ICOS}^{+} \mathrm{CD} 4 \mathrm{~T}$-cells measured by flow cytometry can be used as a reproducible pharmacodynamic biomarker to indicate biologic activity in the setting of}

\footnotetext{
${ }^{\dagger}$ Correspondence: Padmanee Sharma, The University of Texas, M.D. Anderson Cancer Center, Box 0018-7, 1515 Holcombe Boulevard, Houston, TX 77030. Phone: 713.792.2830; Fax: 713.745.1625; padsharma@ mdanderson.org.

Conflict of Interest Disclosure: P.S., J.D.W., and J.P.A. have served on BMS advisory boards and received honorariums for their services. JPA is the inventor of intellectual property owned by the University of California, Berkeley, and has received royalties from BMS.
} 
anti-CTLA-4 therapy, which should enable appropriate immune monitoring to determine whether patients receiving anti-CTLA-4 monotherapy or combination treatment strategies are having an adequate biologic response.

\section{Keywords}

T-cells; immunotherapy; anti-CTLA-4; biomarker; ICOS

\section{Introduction}

Cytotoxic lymphocyte antigen-4 (CTLA-4) is an inhibitory co-receptor expressed on T-cells and acts to attenuate T-cell immune responses (1-3). Blockade of CTLA-4 with a monoclonal antibody $(\mathrm{mAb})$ has been shown to enhance $\mathrm{T}$-cell responses and induce tumor rejection in a number of animal models $(4,5)$. Ipilimumab (Bristol-Myers Squibb, BMS), a monoclonal antibody to human CTLA-4, has been found to elicit objective responses in clinical trials (6-10) and was shown to improve median overall survival of patients with metastatic melanoma in two different Phase III clinical trials $(11,12)$.

In a pre-surgical clinical trial, in which anti-CTLA-4 (ipilimumab) was administered to patients with localized bladder cancer, we previously reported a significant increase in the frequency of T-cells expressing the inducible costimulator (ICOS) molecule in both tumor tissues and blood samples obtained from patients. $(13,14)$ We subsequently reported that a sustained increase in the frequency of $\mathrm{ICOS}^{+} \mathrm{CD} 4 \mathrm{~T}$-cells correlated with clinical benefit in a small cohort of patients with melanoma who received anti-CTLA-4 therapy (15). We also found that loss of the ICOS/ICOS-ligand pathway in a murine model led to impaired antitumor immune responses and tumor rejection (16). Another study, with a different antiCTLA-4 antibody (tremelimumab, Pfizer) also reported an increase in $\mathrm{ICOS}^{+}$CD4 T-cells in blood samples obtained from patients with breast cancer (17). Collectively, these data indicated a biologically relevant role for ICOS in anti-tumor responses elicited by antiCTLA-4 therapy.

ICOS is a T-cell specific molecule that belongs to the CD28/CTLA-4 family $(18,19)$ and is expressed only after T-cell activation. Since anti-CTLA-4 enhances T-cell activation, we hypothesized that ICOS expression on CD4 T-cells could be used as a pharmacodynamic biomarker for anti-CTLA-4 therapy. To determine whether ICOS expression on CD4 T-cells measured by flow cytometry could be used as a pharmacodynamic biomarker for antiCTLA-4 therapy (ipilimumab), we analyzed blood samples from 56 individuals consisting of 10 healthy donors, 36 patients who received ipilimumab (12 patients with bladder cancer and 24 patients with melanoma), and 10 patients with melanoma who received treatment with a different immunomodulatory agent (gp100 DNA vaccine).

\section{Materials and Methods}

\section{Normal healthy donors and cancer patients}

Blood samples were collected after normal healthy donors provided informed consent on M. D. Anderson Cancer Center IRB-approved lab protocol 2005-0027. Blood samples from 
cancer patients were obtained after they provided consent on IRB-approved protocols such that patients with bladder cancer were consented as per protocols 2006-0080 and 2005-0027, as previously published $(13,15)$, and patients with melanoma were either consented on the Memorial Sloan-Kettering Cancer Center IRB-approved biospecimen collection protocols 95-090 or 00-144 prior to treatment with ipilimumab (NCT00495066), as previously published (15), or protocol 06-113 for treatment with gp100 DNA vaccine (NCT00398073), as previously published (20).

\section{Blood processing}

All blood samples were collected from the clinic and transported at room temperature within 3 hours after being drawn from each individual. Whole blood was collected in Vacutainer tubes containing sodium heparin (BD Vacutainer). Peripheral blood mononuclear cells (PBMCs) were isolated from whole blood by density gradient centrifugation using Lymphocyte Separation Medium (Mediatech) and Leucosep tubes (Greiner Bio-one). In brief, whole blood was centrifuged at $410 \mathrm{~g}$ for 10 minutes and the plasma layer was collected, centrifuged for 10 minutes at $885 \mathrm{~g}$, and the supernatant frozen at $-80^{\circ} \mathrm{C}$ for subsequent experiments. The whole blood was diluted 1:1 with RPMI (Mediatech) and the PMBCs isolated per manufacturer's guidelines. Cells at the interface were harvested and washed once with RPMI and red blood cells were lysed with ammonium chloride lysis buffer. Cells were washed, counted, and immediately used fresh for staining and flow cytometry analysis as previously described (13) or 10 million PBMCs were frozen per ml of freezing solution (10\% DMSO 10\% FBS supplemented RPMI) per NUNC cryovial. PBMCs were frozen in a Nalgene Cryo $1^{\circ} \mathrm{C}$ freezing container per manufacturer's instructions and stored in liquid nitrogen thereafter.

\section{In vitro activation}

PBMCs were resuspended at $1.5 \times 10^{6}$ cells $/ \mathrm{ml}$ in RPMI (Mediatech) supplemented with MEM non-essential amino acids, $2 \mathrm{mM}$ L-glutamine, 100U/ml penicillin, $100 \mathrm{ug} / \mathrm{ml}$ streptomycin, and 10\% human AB serum (Life Technologies); $30 \times 10^{6}$ PBMCs were added to appropriate T25 tissue culture flask (Corning). PMBCs were activated for 48 hours with immobilized anti-CD3 5ug/ml clone UCHT1 and soluble anti-CD28 2ug/ml clone CD28.2 (BD Biosciences). PBMCs were collected after detachment from the flask with $4 \mathrm{mM}$ NaEDTA, washed twice with PBS $+2 \%$ FBS, followed immediately by labeling for flow cytometry or freezing as above for approximately 24 hours before labeling for flow cytometry.

\section{Flow Cytometry}

Antibodies used for flow cytometry analysis were CD4 PerCP Cy5.5 (BD Biosciences) and ICOS PE-Cy7 (eBioscience), and FoxP3-PE. Lineage was excluded using a cocktail of FITC conjugated antibodies, CD303 (Miltenyi Biotec), CD20, CD16, CD14, CD56, TCR $\gamma \delta$ and CD19 (BD Biosciences). Triplicate samples were analyzed using the FACSCanto II (Becton Dickinson). Data were analyzed using the BD FACSDiva software. Gates were set according to appropriate isotype controls. DNT and JS conducted studies independently. 


\section{Statistical Analyses}

Summary statistics such as mean, median, standard deviation and $95 \%$ confidence interval were provided for the frequency of $\mathrm{CD}^{+}{ }^{+} \mathrm{ICOS}^{+} \mathrm{T}$-cells. The histograms and bar plots were used to display ICOS expressions by different subject group. Wilcoxon signed rank test for paired data was used to assess the difference on the frequency of $\mathrm{ICOS}^{+} \mathrm{CD} 4 \mathrm{~T}$-cells between prior and post treatment. Wilcoxon rank sum test was also used to assess the difference on the frequency of $\mathrm{ICOS}^{+} \mathrm{CD} 4 \mathrm{~T}$-cells at baseline between donor controls and patients with different tumor types. All p-values presented are two-sided. P-values less than 0.05 were considered to be statistically significant. Statistical analyses were carried out using SPLUS 7 (Insightful Corp, Seattle, WA)

\section{Results \\ ICOS expression on CD4 T-cells was consistently increased after patients received treatment with anti-CTLA-4}

In a subset of 3-6 bladder cancer patients who received 2 doses of anti-CTLA-4 and for whom PBMCs were available, we evaluated multiple potential pharmacodynamic biomarkers including: expression of ICOS, HLA-DR, and CD25 on CD4 T-cells; frequency of total CD4 and CD8 T-cell populations; and absolute lymphocyte count. As shown in Table 1, an increased frequency of $\mathrm{ICOS}^{+} \mathrm{CD} 4 \mathrm{~T}$-cells was consistently found after patients received treatment with dose \#1 and dose \#2 of anti-CTLA-4. However, we did not observe a consistent change in the other 5 evaluated biomarkers after patients received treatment with 2 doses of anti-CTLA-4. These data suggested that ICOS expression on CD4 T-cells was a reasonable biomarker to further pursue as a potential pharmacodynamic biomarker for anti-CTLA-4 therapy.

\section{Flow cytometric studies can reproducibly measure frequency of ICOS ${ }^{+}$CD4 T-cells}

Since we found that anti-CTLA-4 (ipilimumab) led to an increased frequency of ICOS ${ }^{+}$CD4 T-cells, which was due to enhanced T-cell activation, we conducted studies to determine whether in vitro activation with anti-CD3 and anti-CD28 would also lead to an increased frequency of $\mathrm{ICOS}^{+} \mathrm{CD} 4 \mathrm{~T}$-cells. We performed in vitro activation with anti-CD3 and antiCD28 on human peripheral blood mononuclear cells (PBMCs) obtained from normal healthy donors in order to determine whether our flow cytometric assay could reliably detect a change in ICOS expression on CD4 T-cells. We found that in vitro activation of human PBMCs led to a detectable increase in $\mathrm{ICOS}^{+} \mathrm{CD} 4 \mathrm{~T}$-cells (Supplemental Figure 1), which was similarly observed for 10 different donors (Supplemental Table 1).

To determine whether our flow cytometric assay was reproducible, we obtained blood samples from 3 healthy donors on 3 different days and evaluated frequency of $\mathrm{ICOS}^{+} \mathrm{CD} 4$ T-cells before and after in vitro activation for both fresh and frozen samples. Blood was obtained from donors on 3 separate days and samples were evaluated by 2 independent operators before and after activation either immediately (fresh) or after being frozen for 24hours (Figure 1, representative donor). Blood samples from 2 additional healthy donors were also obtained on 3 separate days and underwent similar analyses (Supplemental Table 2). Each donor sample was tested in triplicate and the coefficient of variation was found to be 
less than $20 \%$ for the two operators (Supplemental Table 2). In addition, we evaluated the intra-assay variability on triplicate samples studied at each timepoint for 6 patients who received treatment with anti-CTLA- 4 and found that the coefficient of variation was less than 10\% (Supplemental Table 3). These data indicated that ICOS expression on CD4 Tcells could be reproducibly detected by flow cytometric analyses.

\section{An increase in the frequency of ICOS ${ }^{+}$CD4 T-cells is specific for anti-CTLA-4 therapy}

To determine whether there were any differences in the frequency of $\mathrm{ICOS}^{+} \mathrm{CD} 4 \mathrm{~T}$-cells in samples obtained from normal healthy donors as compared to baseline (pre-therapy) samples obtained from cancer patients, we compared data from a cohort of normal healthy donors $(\mathrm{n}=10)$ to pre-therapy data obtained from patients with localized bladder cancer $(\mathrm{n}=12)$ and patients with metastatic melanoma $(n=34 ; 24$ patients who would receive treatment with ipilimumab and 10 patients who would receive treatment with gp100 DNA vaccine). As shown in Table 2, there was not a statistically significant difference in the frequency of $\mathrm{ICOS}^{+} \mathrm{CD} 4 \mathrm{~T}$-cells at baseline among these cohorts. However, after treatment with antiCTLA-4, the frequency of $\mathrm{ICOS}^{+}$CD4 T-cells significantly increased in patients with localized bladder cancer and metastatic melanoma ( $\mathrm{p} \leq 0.004$ ). Patients who were treated with a different immunomodulatory agent, gp100 DNA vaccine, did not demonstrate a statistically significant change in the frequency of $\operatorname{ICOS}^{+} \mathrm{CD} 4 \mathrm{~T}$-cells $(\mathrm{p}=0.92)$ (Table 3). We used the upper bound of $95 \%$ confidence interval obtained from the baseline samples $(\mathrm{n}=56)$ to define a positive test for the frequency of $\mathrm{ICOS}^{+} \mathrm{CD} 4 \mathrm{~T}$-cells as greater than 5.63. Based on this threshold, flow cytometric analysis for the frequency of $\mathrm{ICOS}^{+} \mathrm{CD} 4 \mathrm{~T}$-cells as a pharmacodynamic biomarker for ipilimumab therapy provided an average specificity of 96\% (95\% confidence interval: 88\%-100\%) and sensitivity of 71\% (95\% CI: 54\%-85\%) after administration of two doses of anti-CTLA-4 (Table 4).

\section{Discussion}

Pharmacodynamic biomarkers are markers of drug effect that usually encompass the pathway associated with the molecular target or represent downstream consequences of target and pathway modulation. In the case of anti-CTLA-4 therapy, the monoclonal antibody blocks the inhibitory CTLA-4 molecule and prevents interactions between CTLA-4 and its ligand (B7), which subsequently enables B7 to be free for interactions with CD28, a critical co-stimulatory molecule for T-cell activation $(21,22)$. Therefore, anti-CTLA-4 therapy leads to enhanced T-cell activation. Upon T-cell activation, ICOS expression is upregulated $(18,23)$; therefore, ICOS can serve as a marker of T-cell activation. We were the first to report that anti-CTLA-4 therapy (ipilimumab) led to an increase in the frequency of ICOS $^{+}$T-cells in cancer patients (13-15). Subsequently, another group found an increase in the frequency of $\mathrm{ICOS}^{+} \mathrm{T}$-cells after cancer patients were treated with a different antiCTLA-4 antibody, tremelimumab (17). In this study, we tested the hypothesis that an increased frequency of ICOS ${ }^{+} \mathrm{CD} 4 \mathrm{~T}$-cells detected by flow cytometry can be used as a pharmacodynamic biomarker for anti-CTLA-4 therapy. We demonstrated 96\% specificity and $71 \%$ sensitivity for flow cytometric measurement of an increased frequency of ICOS ${ }^{+}$ CD4 T-cells after treatment with anti-CTLA-4. 
Although we present data to show that the frequency of $\mathrm{ICOS}^{+} \mathrm{CD} 4 \mathrm{~T}$-cells can serve as a pharmacodynamic biomarker for anti-CTLA-4 therapy, it should be noted that ICOS ${ }^{+} \mathrm{T}$ cells also play an important role in the anti-tumor response mediated by anti-CTLA-4 therapy. We have previously shown that mice lacking ICOS or its ligand (ICOSL) have impaired anti-tumor responses after treatment with anti-CTLA-4, as compared to wild-type mice (16). Therefore, based on the clear relationship between T-cell activation and upregulation of ICOS expression, as well as the biologic role of the ICOS/ICOSL pathway in mediating anti-tumor immune responses, we propose that ICOS expression on CD4 Tcells represents a valid biomarker to be further developed in the setting of anti-CTLA-4 therapy.

Anti-CTLA-4 (ipilimumab) is currently FDA-approved for the treatment of patients with metastatic melanoma. In addition, since anti-CTLA-4 targets a T-cell specific molecule, as opposed to a tumor-specific molecule, it is being tested in multiple clinical trials in patients with different malignancies, which will require appropriate development of biomarkers for anti-CTLA-4 therapy. Also, since pre-clinical data suggest that combination therapies with agents that prime a T-cell response plus anti-CTLA-4 are more effective at eliciting antitumor responses as compared to monotherapy (24-26), clinical trials are underway with antiCTLA-4 in various combination strategies, which will require appropriate biomarker development to evaluate whether the combination maintains biologic efficacy of antiCTLA-4. Given the number of clinical trials that are ongoing or are expected to be conducted with anti-CTLA-4 antibody, appropriate biomarkers are clearly needed to enable rational decisions regarding dose, schedule and combinations. Here, we define an increased frequency of $\mathrm{ICOS}^{+} \mathrm{CD} 4 \mathrm{~T}$-cells measured by flow cytometry as a pharmacodynamic biomarker that can be measured in blood samples, which can potentially be used for making decisions regarding different doses, schedules and/or combinations tested in clinical trials with anti-CTLA-4. Our work represents a small retrospective analysis and warrants further prospective studies in a larger cohort of patients.

\section{Supplementary Material}

Refer to Web version on PubMed Central for supplementary material.

\section{Acknowledgments}

Grant Support: This work was supported in part by a UTMDACC Physician-Scientist Program Award, a Career Development Award from the UTMDACC Bladder Cancer SPORE, a Melanoma Research Alliance Young Investigator Award and a Doris Duke Clinical Scientist Development Award (all to P.S.). Bristol-Myers Squibb (BMS) sponsored and funded the clinical trials with Ipilimumab.

\section{References}

1. Walunas TL, Lenschow DJ, Bakker CY, Linsley PS, Freeman GJ, Green JM, et al. CTLA-4 can function as a negative regulator of T-cells activation. Immunity. 1994; 1:405-13. [PubMed: 7882171]

2. Krummel MF, Allison JP. CD28 and CTLA-4 have opposing effects on the response of T-cells to stimulation. J Exp Med. 1995; 182:459-65. [PubMed: 7543139]

3. Krummel MF, Allison JP. CTLA-4 engagement inhibits IL-2 accumulation and cell cycle progression upon activation of resting T-cells. J Exp Med. 1996; 183:2533-40. [PubMed: 8676074] 
4. Leach DR, Krummel MF, Allison JP. Enhancement of antitumor immunity by CTLA-4 blockade. Science. 1996; 271:1734-36. [PubMed: 8596936]

5. van Elsas A, Hurwitz AA, Allison JP. Combination immunotherapy of B16 melanoma using anticytotoxic T lymphocyte-associated antigen 4 (CTLA-4) and granulocyte/macrophage colonystimulating factor (GM-CSF)-producing vaccines induces rejection of subcutaneous and metastatic tumors accompanied by autoimmune depigmentation. J Exp Med. 1999; 190:355-66. [PubMed: 10430624]

6. Hodi FS, Mihm MC, Soiffer RJ, Haluska FG, Butler M, Seiden MV, et al. Biologic activity of cytotoxic T lymphocyte-associated antigen 4 antibody blockade in previously vaccinated metastatic melanoma and ovarian carcinoma patients. Proc Natl Acad Sci USA. 2003; 100:4712-17. [PubMed: 12682289]

7. Phan GQ, Yang JC, Sherry RM, Hwu P, Topalian SL, Schwartzentruber DJ, et al. Cancer regression and autoimmunity induced by cytotoxic T lymphocyte-associated antigen 4 blockade in patients with metastatic melanoma. ProcNatl Acad Sci USA. 2003; 100:8372-77.

8. Korman A, Yellin M, Keler T. Tumor immunotherapy: preclinical and clinical activity of antiCTLA-4 antibodies. Curr Opin Investig Drugs. 2005; 6:582-91.

9. Saenger YM, Wolchok JD. The heterogeneity of the kinetics of response to ipilimumab in metastatic melanoma: Patient cases. Cancer Immun n. 2008; 8:1-7.

10. Small EJ, Tchekmedyian S, Rini BI, Fong L, Lowy I, Allison JP. A pilot trial of CTLA-4 blockade with human anti-CTLA-4 in patients with hormone-refractory prostate cancer. Clin Cancer Res. 2007; 13:1810-15. [PubMed: 17363537]

11. Hodi FS, O'Day SJ, McDermott DF, Weber RW, Sosman JA, Haanen JB, et al. Improved survival with Ipilimumab in patients with metastatic melanoma. N Engl J Med. 2010; 363:711-23. [PubMed: 20525992]

12. Robert C, Thomas L, Bondarenko I, O'Day S, Weber J, Garbe C, et al. Ipilimumab plus Dacarbazine for previously untreated metastatic melanoma. N Engl J Med. 2011; 364:2517-26. [PubMed: 21639810]

13. Liakou CI, Kamat A, Ng Tang D, Chen H, Sun J, Troncoso P, et al. CTLA-4 blockade increases IFN $\gamma$-producing $\mathrm{CD}^{+}{ }^{+} \mathrm{ICOS}^{\text {hi }}$ cells to shift the ratio of effector to regulatory T-cells in cancer patients. Proc Natl Acad Sci USA. 2008; 105:14987-92. [PubMed: 18818309]

14. Chen H, Liakou CI, Kamat A, Pettaway C, Ward JF, Ng Tang D, et al. Anti-CTLA-4 therapy results in higher $\mathrm{CD} 4{ }^{+} \mathrm{ICOS}^{\text {hi }}$ T-cells frequency and IFN- $\gamma$ levels in both nonmalignant and malignant prostate tissues. ProcNatl Acad Sci USA. 2009; 106:2729-34.

15. Carthon BC, Wolchok JD, Yuan J, Kamat A, Ng Tang DS, Sun J, et al. Preoperative CTLA-4 blockade: Tolerability and immune monitoring in the setting of a presurgical clinical trial. Clin Cancer Res. 2010; 16:2861-71. [PubMed: 20460488]

16. Fu T, He Q, Sharma P. The ICOS/ICOSL pathway is required for optimal antitumor responses mediated by anti-CTLA-4 therapy. Cancer Res. 2011; 71:5445-54. [PubMed: 21708958]

17. Vonderheide RH, LoRusso PM, Khalil M, Gartner EM, Khaira D, Soulieres D, et al. Tremelimumab in combination with Exemestane in patients with advance breast cancer and treatment-associated modulation of inducible costimulator expression on patient T-cellss. Clin Cancer Res. 2010; 16:3485-94. [PubMed: 20479064]

18. Hutloff A, Dittrich AM, Beier KC, Eljaschewitsch B, Kraft R, Anagnostopoulos I, et al. ICOS is an inducible T-cells co-stimulator structurally functionally related CD28. Nature. 1999; 397:263-66. [PubMed: 9930702]

19. Dong C, Juedes AE, Temann U, Shresta S, Allison JP, Ruddle NH, et al. ICOS co stimulatory receptor is essential for T-cells activation and function. Nature. 2001; 409:97-101. [PubMed: $11343121]$

20. Ginsberg BA, Gallardo HF, Rasalan TS, Adamow M, Mu Z, Tandon S, et al. Immunologic Response to Xenogeneic gp100 DNA in Melanoma Patients: Comparison of Particle-Mediated Epidermal Delivery with Intramuscular Injection. Clin Cancer Res. 2010; 16:4057-65. [PubMed: 20647477]

21. Lenschow DJ, Walunas TL, Bluestone JA. CD28/B7 System of T-cells co-stimulation. Annu Rev Immunol. 1996; 14:233-58. [PubMed: 8717514] 
22. Chambers CA, Allison J P. Co-stimulatory regulation of T-cells function. Curr Opin Cell Biol. 1999; 11:203-10. [PubMed: 10209159]

23. Yoshinaga SK, Whoriskey JS, Khare SD, Sarmiento U, Guo J, Horan T, et al. T-cells costimulation through B7RP-1 and ICOS. Nature. 1999; 402:827-32. [PubMed: 10617205]

24. Hurwitz AA, Foster BA, Kwon ED, Truong T, Choi EM, Greenberg NM, et al. Combination immunotherapy of primary prostate cancer in a transgenic mouse model using CTLA-4 blockade. Cancer Res. 2000; 60:2444-48. [PubMed: 10811122]

25. Quezada SA, Peggs KS, Curran MA, Allison JP. CTLA4 blockade and GM-CSF combination immunotherapy alters the intratumor balance of effector and regulatory T-cells. J Clin Invest. 2006; 116:1935-45. [PubMed: 16778987]

26. Dewan MZ, Galloway AE, Noriko Kawashima N, Dewyngaert JK, Babb JS, Formenti SC, et al. Fractionated but not single-dose radiotherapy induces an immune-mediated abscopal effect when combined with anti-CTLA-4 antibody. Clin Cancer Res. 2009; 15:5379-88. [PubMed: 19706802] 


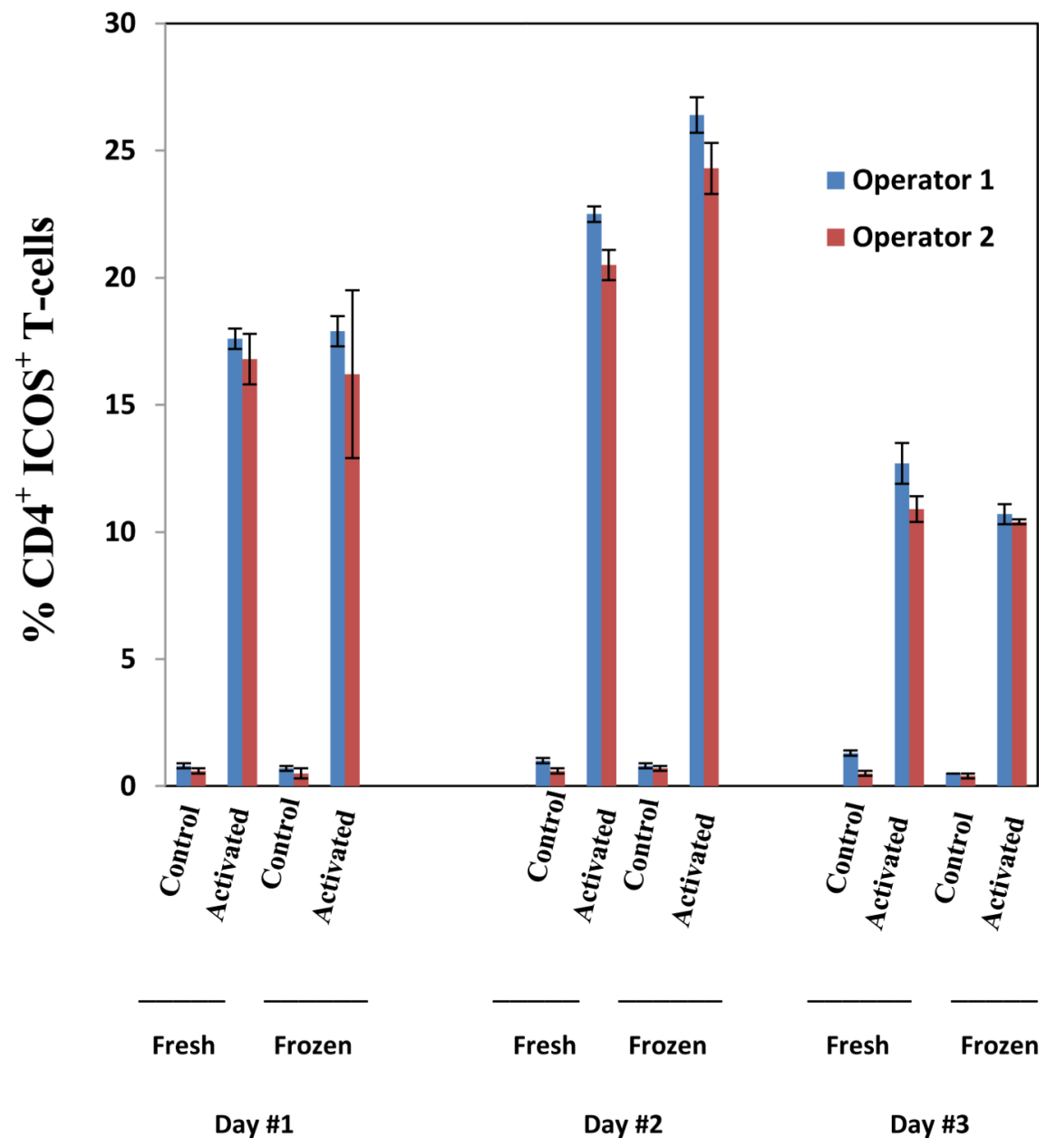

Figure 1. Flow cytometric studies can reproducibly measure frequency of $\mathrm{ICOS}^{+} \mathrm{CD} 4 \mathrm{~T}$-cells Peripheral blood from a single individual was drawn on 3 different days and cells were unactivated (control) or activated in vitro prior to 2 different operators performing flow cytometry to evaluate frequency of $\mathrm{ICOS}^{+} \mathrm{CD} 4 \mathrm{~T}$-cells on fresh or frozen samples. 
Table 1

Evaluation of 6 different immunologic parameters in patients treated with anti-CTLA-4

\begin{tabular}{|c|c|c|c|}
\hline \multicolumn{4}{|c|}{ Patients with bladder cancer who received anti-CTLA- 4 therapy } \\
\hline \multicolumn{4}{|c|}{$\% \mathrm{ICOS}^{+} \mathrm{CD} 4$ T-cells } \\
\hline Patient & Pre-treatment & Post-treatment (dose \#1) & Post-treatment (dose \#2) \\
\hline 1 & 1.4 & 13.5 & 8.7 \\
\hline 2 & 1.9 & 9.7 & 21.7 \\
\hline 3 & 2.8 & 9.7 & 40.1 \\
\hline 4 & 1.9 & 14.2 & 6.4 \\
\hline 5 & 4.2 & 16.6 & 27.8 \\
\hline 6 & 2.9 & 13.5 & 6.6 \\
\hline
\end{tabular}

\begin{tabular}{|c|c|c|c|}
\hline \multicolumn{4}{|c|}{ \% HLA-DR ${ }^{+}$CD4 T-cells } \\
\hline Patient & Pre-treatment & Post-treatment (dose \#1) & Post-treatment (dose \#2) \\
\hline 1 & 3.5 & 6.5 & 3.4 \\
\hline 2 & 1.2 & 3.0 & 1.2 \\
\hline 3 & 1.9 & 8.3 & 5.2 \\
\hline
\end{tabular}

\begin{tabular}{|c|c|c|c|}
\hline \multicolumn{3}{|c|}{ \% CD25 $^{+}$CD4 T-cells } \\
\hline Patient & Pre-treatment & Post-treatment (dose \#1) & Post-treatment (dose \#2) \\
\hline 1 & 6.8 & 10.9 & 18.8 \\
\hline 2 & 30.7 & 31.3 & 38.5 \\
\hline 3 & 43.0 & 37.4 & 38.5 \\
\hline
\end{tabular}

\% CD4 T-cells

\begin{tabular}{|c|c|c|c|}
\hline Patient & Pre-treatment & Post-treatment (dose \#1) & Post-treatment (dose \#2) \\
\hline 1 & 52.7 & 51.3 & 51.5 \\
\hline 2 & 32.1 & 40.5 & 43.5 \\
\hline 3 & 41.0 & 37.1 & 23.7 \\
\hline 4 & 46.9 & 45.0 & 45.4 \\
\hline 5 & 22.4 & 41.7 & 35.3 \\
\hline 6 & 29.6 & 35.7 & 34.3 \\
\hline
\end{tabular}

\begin{tabular}{|c|c|c|c|}
\hline \multicolumn{4}{|c|}{ \% CD8 T-cells } \\
\hline Patient & Pre-treatment & Post-treatment (dose \#1) & Post-treatment (dose \#2) \\
\hline 1 & 11.5 & 6.7 & 10.8 \\
\hline 2 & 12.0 & 15.3 & 22.7 \\
\hline 3 & 11.5 & 10.5 & 5.8 \\
\hline 4 & 16.1 & 13.2 & 26.0 \\
\hline 5 & 6.1 & 6.8 & 5.8 \\
\hline 6 & 9.3 & 8.9 & 7.5 \\
\hline
\end{tabular}




\begin{tabular}{|c|c|c|c|}
\hline \multicolumn{4}{|c|}{ Absolute lymphocyte count (k/ul) } \\
\hline Patient & Pre-treatment & Post-treatment (dose \#1) & Post-treatment (dose \#2) \\
\hline 1 & 2.34 & 3.17 & 1.86 \\
\hline 2 & 3.51 & 4.63 & 5.13 \\
\hline 3 & 1.88 & 1.91 & 0.95 \\
\hline 4 & 3.94 & 2.82 & 4.49 \\
\hline 5 & 0.76 & 1.13 & 1.08 \\
\hline 6 & 0.90 & 2.83 & 3.21 \\
\hline
\end{tabular}




\section{Table 2}

Frequency of $\mathrm{ICOS}^{+} \mathrm{CD} 4 \mathrm{~T}$-cells from normal healthy donors and baseline blood samples from cancer patients

\begin{tabular}{|c|c|c|c|c|c|}
\hline & All Samples* & Healthy donors & $\begin{array}{c}\text { Cancer Patients (Localized } \\
\text { bladder cancer, will receive } \\
\text { ipilimumab therapy) }\end{array}$ & $\begin{array}{c}\text { Cancer Patients } \\
\text { (Metastatic melanoma, } \\
\text { will receive ipilimumab } \\
\text { therapy) }\end{array}$ & $\begin{array}{c}\text { Cancer Patients } \\
\text { (Metastatic melanoma, will } \\
\text { receive gp100 vaccine } \\
\text { therapy) }\end{array}$ \\
\hline $\mathrm{N}$ & $56^{*}$ & 10 & 12 & 24 & 10 \\
\hline median & 2 & 2 & 2.5 & 1 & 2 \\
\hline mean & 2.22 & 1.90 & 2.36 & 2.08 & 2.7 \\
\hline SD & 1.39 & 0.99 & 1.18 & 1.67 & 1.25 \\
\hline p-value & -- & reference & 0.323 & 0.737 & 0.128 \\
\hline
\end{tabular}


Table 3

Frequency of $\mathrm{ICOS}^{+} \mathrm{CD} 4 \mathrm{~T}$-cells in pre- and post- therapy samples after treatment with anti-CTLA-4

\begin{tabular}{|l|c|c|c|c|c|c|c|c|c|c|}
\hline & \multicolumn{3}{|c|}{$\begin{array}{c}\text { Patients with localized bladder } \\
\text { cancer (ipilimumab therapy) }\end{array}$} & \multicolumn{2}{c|}{$\begin{array}{c}\text { Patients with metastatic } \\
\text { melanoma (ipilimumab therapy) }\end{array}$} & \multicolumn{3}{|c|}{$\begin{array}{c}\text { Patients with metastatic } \\
\text { melanoma (gp100 DNA vaccine) }\end{array}$} \\
\hline & Mean & SD & p-value & Mean & SD & p-value & Mean & SD & p-value \\
\hline pre-treatment & 2.36 & 1.18 & Ref. & 2.08 & 1.67 & Ref. & 2.70 & 1.25 & Ref. \\
\hline post-treatment (dose 1) & 13.33 & 4.14 & 0.003 & 6.13 & 5.27 & 0.001 & 2.70 & 0.95 & 0.92 \\
\hline post-treatment (dose 2) & 16.27 & 10.29 & 0.004 & 7.46 & 6.18 & 0.0001 & & & \\
\hline
\end{tabular}


Table 4

Sensitivity and specificity of $\mathrm{ICOS}^{+} \mathrm{CD} 4$ T-cells as a pharmacodynamic biomarker of anti-CTLA-4 therapy

\begin{tabular}{|l|c|c|c|c|c|}
\hline & & $\mathbf{N}$ & Greater than $\mathbf{5 . 6 3}{ }^{*}(+)$ & Sensitivity (95\% CI) & Specificity (95\% CI) \\
\hline Pre-treatment & All samples & 56 & 2 & -- & $96.4 \%(87.7 \%, 99.6 \%)$ \\
\hline After dose \#2 of ipilimumab & Cancer Patients & 35 & 25 & $71 \%(53.7 \%, 85.4 \%)$ & - \\
\hline
\end{tabular}

* upper bound of $95 \%$ confidence interval from baseline samples 